\section{Discussion}

Transcatheter occlusion techniques have become an increasingly used alternative to surgical closure of the ASD, and various devices are available for this. ${ }^{1-3}$ Comparison of transcatheter and surgical closure of secundum ASD in children and adults reveals a lower complication rate in the device closure group. ${ }^{3,4}$ Moreover, in patients experiencing unsatisfactory device position, retrieval is usually feasible at the time of implantation, followed by elective surgical closure. If embolization (for which transcatheter retrieval is unsuccessful) occurs, urgent surgical therapy is necessary. ${ }^{2,3,5}$

The most important reason for acute failure of these devices is poor patient selection, device selection, or both. ${ }^{1,5}$ Other suggested mechanisms are device-related failure, inadequate experience, ${ }^{2,5}$ poor defect rim to hold the device, ${ }^{1-3}$ and tearing of the interatrial septum caused by catheter and device manipulation. ${ }^{2,5}$ A part or the entire device might embolize to the right or left atrium, main pulmonary artery, or even to other parts of the vascular tree on both the right and left sides of the circulation. Once the device is detached from its cable, it becomes difficult to retrieve, and depending on its location, it might even become lethal.

Although transcatheter devices represent useful alternatives to surgical closure in selected cases, they can be associated with failures and, most importantly, with life-threatening complications. Thus proper selection of patient and device is mandatory. Close monitoring and facilities for emergency surgical intervention should be available for all patients.

\section{References}

1. Berger F, Vogel M, Alexi-Meskishvili V, Lange P. Comparison of results and complications of surgical and Amplatzer device closure of atrial septal defects. J Thorac Cardiovasc Surg. 1999;118:674-80.

2. Chessa M, Carminatti M, Butera G, Binni RM, Drago M, Rosti L, et al. Early and late complications associated with transcatheter occlusion of atrial septal defect. J Am Coll Cardiol. 2002;39:1061-5.

3. Thomson J, Aburawi E, Watterson K, Van Doorn C, Gibbs J. Surgical and transcatheter (Amplatzer) closure of atrial septal defects: a prospective comparison of results and cost. Heart. 2002;87:466-9.

4. Du Zhong-Dong, Hijazzi Z, Kleinman C, Silverman N, Larntz K. Comparison between transcatheter and surgical closure of secundum atrial septal defect in children and adults. J Am Coll Cardiol. 2002;39: 1836-44.

5. Agarwal SK, Ghosh P, Mittal P. Failure of devices used for closure of atrial septal defects: mechanisms and management. $J$ Thorac Cardiovasc Surg. 1996;112:21-6.

\title{
Collision of the caval flows caused early failure of the Fontan circulation
}

\author{
Hirohisa Murakami, MD, ${ }^{a}$ Naoki Yoshimura, MD, ${ }^{a}$ Junichiro Kitahara, MD, ${ }^{a}$ Shingo Otaka, MD, ${ }^{a}$ Fikiko Ichida, MD, \\ and Takuro Misaki, MD, ${ }^{a}$ Toyama, Japan
}

I n 1988 , de Leval and colleagues ${ }^{1}$ demonstrated the importance of fluid dynamics in Fontan circulation. They developed the total cavopulmonary connection (TCPC), which has hemodynamic advantages compared with the traditional atriopulmonary connection. In regard to the hemodynamics of the cavopulmonary anastomosis, several studies demonstrated that collision of caval flows at the connection resulted in high dissipative energy losses and that introduction of an offset between the superior vena cava (SVC) and the inferior vena cava (IVC) inlets reduced those losses. ${ }^{2,3} \mathrm{We}$ describe an early failure of the Fontan circulation caused by collision of the caval flows.

From the First Department of Surgery and Pediatrics, ${ }^{\mathrm{b}}$ Faculty of Medicine, University of Toyama, Toyama, Japan.

Received for publication June 23, 2006; accepted for publication Aug 11, 2006.

Address for reprints: Hirohisa Murakami, MD, First Department of Surgery, Faculty of Medicine, University of Toyama, Toyama 2630, Sugitani, Toyama, 930-0194, Japan (E-mail: murahisa@med.u-toyama.ac.jp).

J Thorac Cardiovasc Surg 2006;132:1235-6

$0022-5223 / \$ 32.00$

Copyright $\odot 2006$ by The American Association for Thoracic Surgery doi:10.1016/j.jtcvs.2006.08.006

\section{Clinical Summary}

The patient was a 2-year-old girl with a diagnosis of tricuspid atresia made soon after birth, for which a bidirectional Glenn (BDG) anastomosis was performed at the age of 7 months. Preoperative catheterization data showed a mean pulmonary artery (PA) pressure of $13 \mathrm{~mm} \mathrm{Hg}$, pulmonary vascular resistance of 3.8 $\mathrm{U} / \mathrm{m}^{2}$, Nakata's PA index of 252 , and left ventricular ejection fraction of 58\%. Angiography showed that mitral valve regurgitation was not present. The site of the BDG anastomosis was deviated to the left side over the vertebra (Figure 1), suggesting that a Fontan-type operation could be safely performed. Two months after the catheterization, extracardiac TCPC was completed with interposition of an expanded polytetrafluoroethylene graft $(\varphi 18 \mathrm{~mm})$ between the right PA and the IVC. After Fontan circulation was established, the patient demonstrated low cardiac output syndrome; her heart rate was 190 beats/min and systolic blood pressure ranged from 50 to $60 \mathrm{~mm} \mathrm{Hg}$. Despite the use of inhaled nitric oxide therapy, the transpulmonary pressure gradient between the central venous pressure (17-19 $\mathrm{mm} \mathrm{Hg}$ ) and the left atrial pressure $(2-3 \mathrm{~mm} \mathrm{Hg})$ increased. Immediate echocardiography showed marked dilatation of the IVC and collapse of the left ventricular cavity. No blood flow was detected in the extracardiac conduit. Urgent angiography was performed. Impingement of SVC flow onto IVC flow and marked stagnation of blood flow in the 


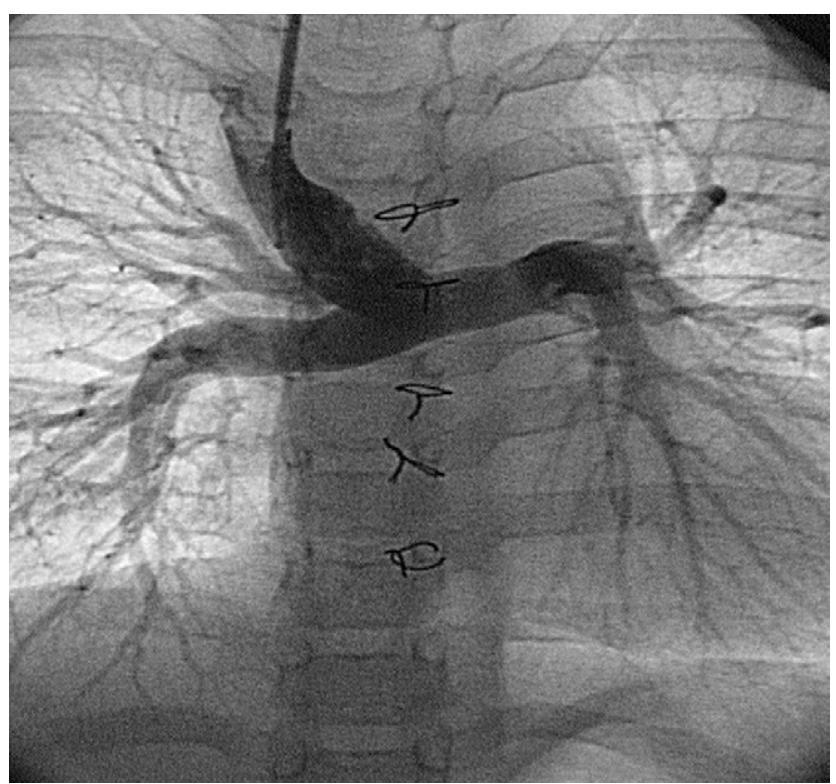

Figure 1. Angiography of the SVC anastomosis to the PAs shows that the BDG is shifted to the left side over the center of the vertebrae.

conduit were seen (Figure 2). Urgent reoperation was performed with cardiopulmonary bypass. After division of the BDG, the anastomosis between the conduit and the right PA was intact, and no thrombus was observed in the conduit through the incision of the PA. The BDG anastomosis was transferred to the right side to avoid significant collision of the caval inlet flows, which was speculated to be the cause of acute failure of the Fontan circulation. Subsequently, the patient's hemodynamics significantly improved. Postoperative echocardiography showed that both the SVC and IVC flows smoothly passed toward the PA. Both the left ventricular volume and the IVC diameter became normal. Although the patient had a severe chylothorax, which required prolonged pleural drainage and a fat-restricted diet, she was discharged in good condition.

\section{Discussion}

A computational simulation model by de Leval and colleagues ${ }^{1}$ demonstrated that flow competition between the SVC and the IVC can occur in TCPC. They also reported a decrease in dissipated power with an increase of at least $1.0 \mathrm{~cm}$ in caval offset. In vitro flow experiments by Sharma and colleagues ${ }^{4}$ showed that as the offset is increased from 0.0 to $0.5 \mathrm{SVC}$ diameters, the power losses decrease significantly. However, there is no report of an actual patient with early failure of the Fontan circulation caused by collision of the SVC and IVC flows. In our case, the addition of an offset dramatically improved the Fontan circulation. Gentles and colleagues $^{5}$ described that unexpected low cardiac output was noted in 20 of 500 patients $(4.0 \%)$ undergoing the Fontan operation. They did not refer to the caval offset; however, early failure

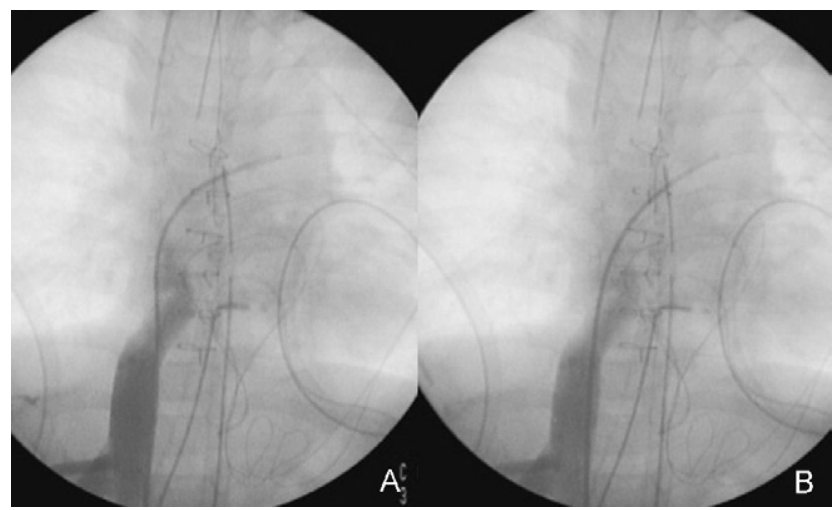

Figure 2. A, Urgent angiography 15 seconds after injection of contrast medium shows the marked delay of blood flow from the conduit to the IVC. The tip of the catheter was located in the left PA, and the contrast medium was washed out immediately by blood flow from the BDG. B, At 30 seconds after injection. The contrast medium was stagnant.

of the Fontan circulation might have been the result of dissipative energy losses of caval return in these patients.

Sharma and colleagues ${ }^{4}$ advocated that surgical strategies for these patients should include a caval offset of 1.0 to 1.5 diameters optimally. Although anatomic space constraints in some clinical situations may allow only a minimal offset, we should try to design an adequate anastomosis to prevent significant energy losses.

\section{Conclusion}

We describe a patient with early failure of the Fontan circulation caused by collision of the caval flows. The addition of caval offset dramatically improved the Fontan circulation. Our clinical experience showed that energy losses in blood flow might be an important factor that may impair the Fontan circulation.

\section{References}

1. de Leval MR, Kilner P, Gewillig M, Bull C. Total cavopulmonary connection: a logical alternative to atriopulmonary connection for complex Fontan operations. J Thorac Cardiovasc Surg. 1988;96:682-95.

2. de Leval MR, Dubini G, Migliavacca F, Jalali H, Camporini G, Redington A, et al. Use of computational fluid dynamics in the design of surgical procedures: application to the study of competitive flows in cavopulmonary connections. J Thorac Cardiovasc Surg. 1996;111: 502-13.

3. Ensley A, Lynch P, Chatzimavroudis G, Lucas C, Sharma S, Yoganathan A. Toward designing the optimal total cavopulmonary connection: an in vitro study. Ann Thorac Surg. 1999;68:1384-90.

4. Sharma S, Goudy S, Walker P, Panchal S, Ensley A, Kanter K, et al. In vitro flow experiments for determination of optimal geometry of total cavopulmonary connection for surgical repair of children with functional single ventricle. J Am Coll Cardiol. 1996;27:1264-9.

5. Gentles TL, Mayer JE, Gauvreau K, Newburger JW, Lock JE, Kupferschmid JP, et al. Fontan operation in five hundred consecutive patients: factors influencing early and late outcome. J Thorac Cardiovasc Surg. 1997;114:376-91. 